MATTERS ARISING

Check for updates

\title{
Reply to 'Sulfisoxazole does not inhibit the secretion of small extracellular vesicles'
}

\author{
Chan-Hyeong Lee ${ }^{1,2}$, Ju-Hyeon Bae ${ }^{1,2}$, Jong-In Kim ${ }^{1,2}$, Ju-Mi Park¹,2, Eun-Ji Choi ${ }^{1,2} \&$ Moon-Chang Baek (D) ${ }^{1 凶}$
}

REPLYING to P. Fonseka et al. Nature Communications https://doi.org/10.1038/s41467-021-21074-x (2021)

$\mathrm{n}$ the previous paper, we showed various evidences that sulfisoxazole (SFX) inhibits the secretion of small extracellular vesicles (sEVs) from MDA-MB231 human breast cell line ${ }^{1}$. The evidences came from the number of sEVs based on nanoparticle tracking analysis (NTA) results, the protein amount of sEVs, and electron microscopy. In addition, we identified the target of SFX, endothelin receptor A (ETA). Inhibitors of ETA, including zibotentan, BQ123, and PD156707, also inhibited the secretion of sEVs from MDA-MB231 cells. Furthermore, the knockdown of ETA with an ETA short hairpin RNA significantly decreased the secretion of sEVs. Collectively, we showed the inhibition of sEV secretion from MDA-MB231 via ETA through pharmacological and genetic approaches. However, Mathivanan's group argues that SFX does not inhibit the secretion of sEVs.

In our original publication, we showed that SFX (Sigma, S6377) inhibits the secretion of sEVs from the MDA-MB231 human breast cell line ${ }^{1}$. In addition, two independent researchers again confirmed the original result with a blind test (with SFX from Sigma, 31739). We also show the inhibition effect of sEV secretion from MDA-MB231 cells by two different SFXs (Sigma, 31739, and manufactured SFX) (Fig. 1). Collectively, these results show that SFX does inhibit the secretion of small extracellular vesicles in our experiments.

The protocol we used to separate sEVs is the same as the published manuscript; supernatants obtained from MDAMB231 cells were serially centrifuged at $300 \times \mathrm{g} / 3 \mathrm{~min}, 2500 \times$ $g / 20 \mathrm{~min}$, and $10,000 \times g / 30 \mathrm{~min}$. In addition, cells were seeded with Dulbecco's modified Eagle's medium (DMEM)/high glucose (HyClone, SH30243.01) with 10\% fetal bovine serum (FBS) (HyClone, SH30084.03), but it was incubated in DMEM/high glucose without FBS when the cells were treated with SFX.

The two points, we thought, should be essentially considered. First is the cytotoxic effect of SFX due to, probably, an unexpected impurity of SFX from different companies. We used SFX concentrations without cytotoxicity in all experiments. If the drug causes cytotoxicity, the number of secreted sEVs might be increased. Second, large amounts of particles still remain in EV-depleted FBS in our laboratory's studies. Therefore, it may be difficult to observe the suppression of $\mathrm{sEV}$ secretion using EV-depleted FBS.

We uploaded detailed materials to the protocol exchange (https://protocolexchange.researchsquare.com).

\section{Methods}

Cells and cell culture. MDA-MB231 cells were obtained from the American Type Culture Collection and grown at $37^{\circ} \mathrm{C}$ under a humidified atmosphere with $5 \% \mathrm{CO}_{2}$ and $95 \%$ air using DMEM (Hyclone) with $10 \%$ FBS (Hyclone) and $1 \%$ antibiotic-antimycotic solution (AA, Hyclone). All cell lines were tested for mycoplasma contamination by polymerase chain reaction genotyping.

Drugs. SFX (31739) was purchased from Sigma-Aldrich. Manufactured SFX was synthesized and provided by Daegu-Gyeongbuk Medical Innovation Foundation, Republic of Korea.

\section{Isolation of SEVs and analysis.}

Step 1: Seed the $5 \times 10^{6}$ cells in 150 -mm plate $(20 \mathrm{ml}$ DMEM with $10 \%$ FBS, $1 \% \mathrm{AA})$.

Step 2: After $24 \mathrm{~h}$, wash using phosphate-buffered saline (PBS) and then change the media (20 $\mathrm{ml}$ DMEM without FBS, AA) with the drug.

Step 3: After $24 \mathrm{~h}$, collect the cell culture supernatant.

Step 4: Centrifuge at $300 \times g, 4^{\circ} \mathrm{C}, 3 \mathrm{~min}$ and transfer the supernatants to a new tube.

Step 5: Centrifuge at $2500 \times g, 4^{\circ} \mathrm{C}, 20 \mathrm{~min}$ and transfer the supernatants to a new tube.

Step 6: Centrifuge at $10,000 \times g, 4^{\circ} \mathrm{C}, 30 \mathrm{~min}$ and transfer the supernatants to a new tube.

Step 7: Filter the supernatants using $0.22-\mu \mathrm{m}$ syringe filters and transfer the supernatants to a new tube (\#344058, Beckman Open-Top Tube).

Step 8: Ultracentrifuge at $120,000 \times g, 4{ }^{\circ} \mathrm{C}, 90 \mathrm{~min}$.

Step 9: Resuspend sEV pellets with $30 \mathrm{ml}$ PBS and centrifuge at $120,000 \times g$ $4{ }^{\circ} \mathrm{C}, 90 \mathrm{~min}$ again.

Step 10: Remove the supernatants and add the $1 \mathrm{ml}$ PBS.

Step 11: After sealing the tube with parafilm, store at $4^{\circ} \mathrm{C}, 1 \mathrm{~h}$ and resuspend the pellet.

Step 12: Transfer the samples to e-tube and analyze using NanoSight and GraphPad Prism.

\footnotetext{
${ }^{1}$ Department of Molecular Medicine, CMRI, Exosome Convergence Research Center (ECRC), School of Medicine, Kyungpook National University, Daegu, Republic of Korea. ${ }^{2}$ These authors contributed equally: Chan-Hyeong Lee, Ju-Hyeon Bae, Jong-In Kim, Ju-Mi Park, Eun-Ji Choi. ${ }^{\bowtie}$ email: mcbaek@knu.ac.kr
} 


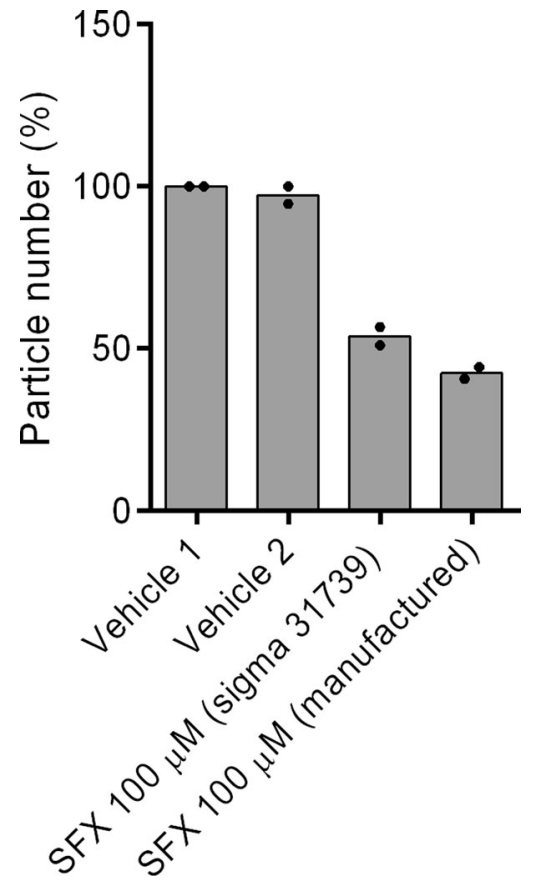

Fig. $1 \mathrm{sEV}$ secretion from MDA-MB-231 cells with or without SFX $100 \mu \mathrm{M}$ (Sigma 31739, and manufactured SFX). All experiments were performed with a blind test ( $n=2$ biologically independent samples).

Reporting summary. Further information on research design is available in the Nature Research Reporting Summary linked to this article.

\section{Data availability}

The detailed protocol has been deposited to the Protocol exchange (https://doi.org/ 10.21203/rs.3.pex-1159/v1). All relevant data of this study are available from the corresponding authors upon reasonable request.

Received: 28 September 2020; Accepted: 13 January 2021;

Published online: 12 February 2021

\section{Reference}

1. Im, E. J. et al. Sulfisoxazole inhibits the secretion of small extracellular vesicles by targeting the endothelin receptor A. Nat. Commun. 10, 1387 (2019).

\section{Acknowledgements}

The new SFX was manufactured by Daegu-Gyeongbuk Medical Innovation Foundation (DGMIF). This research was supported by the Bio \& Medical Technology Development Program of the National Research Foundation (NRF) of Korea funded by the Ministry of Science \& ICT (2017M3A9G8083382).

\section{Author contributions}

C.-H.L. designed, performed, and analyzed experiments, and wrote the manuscript. J.-I.K., E.-J.C., and J.-M.P. performed sample preparation and analysis. J.-H.B. performed NTA analysis. M.-C.B. conceived the idea, supervised the study, designed experiments, and wrote the manuscript.

\section{Competing interests}

The authors declare no competing interests.

\section{Additional information}

Supplementary information The online version contains supplementary material available at https://doi.org/10.1038/s41467-021-21075-w.

Correspondence and requests for materials should be addressed to M.-C.B.

Reprints and permission information is available at http://www.nature.com/reprints

Publisher's note Springer Nature remains neutral with regard to jurisdictional claims in published maps and institutional affiliations.

\begin{abstract}
Open Access This article is licensed under a Creative Common Attribution 4.0 International License, which permits use, sharing, adaptation, distribution and reproduction in any medium or format, as long as you give appropriate credit to the original author(s) and the source, provide a link to the Creative Commons license, and indicate if changes were made. The images or other third party material in this article are included in the article's Creative Commons license, unless indicated otherwise in a credit line to the material. If material is not included in the article's Creative Commons license and your intended use is not permitted by statutory regulation or exceeds the permitted use, you will need to obtain permission directly from the copyright holder. To view a copy of this license, visit http://creativecommons.org/ licenses/by/4.0/.
\end{abstract}

(C) The Author(s) 2021 